\title{
Responses of River Runoff to Climate Change Based on Nonlinear Mixed Regression Model in Chaohe River Basin of Hebei Province, China
}

\author{
JIANG Yan ${ }^{1,2}$, LIU Changming ${ }^{2,3}$, ZHENG Hongxing ${ }^{3}$, LI Xuyong $^{1}$,WU Xianing ${ }^{4}$ \\ (1. State Key Laboratory of Urban and Regional Ecology, Research Center for Eco-Environmental Sciences, Chinese Academy of \\ Sciences, Beijing 100085, China; 2. College of Water Sciences, Beijing Normal University, Beijing 100875, China; \\ 3. Institute of Geographic Sciences and Natural Resources Research, Chinese Academy of Sciences, \\ Beijing 100101, China; 4. Sinohydro Corporation Limited, Beijing 100044, China)
}

\begin{abstract}
Taking the nonlinear nature of runoff system into account, and combining auto-regression method and multi-regression method, a Nonlinear Mixed Regression Model (NMR) was established to analyze the impact of temperature and precipitation changes on annual river runoff process. The model was calibrated and verified by using BP neural network with observed meteorological and runoff data from Daiying Hydrological Station in the Chaohe River of Hebei Province in 1956-2000. Compared with auto-regression model, linear multi-regression model and linear mixed regression model, NMR can improve forecasting precision remarkably. Therefore, the simulation of climate change scenarios was carried out by NMR. The results show that the nonlinear mixed regression model can simulate annual river runoff well.
\end{abstract}

Keywords: river runoff; runoff forecast; nonlinear mixed regression model; linear multi-regression model; linear mixed regression model; BP neural network

\section{Introduction}

Climate change is now widely recognized as the major environmental problem facing the globe (Science, 1977). Therefore, it is important to study the impacts of climate change on hydrological system and water resources system, which can provide the fundamental basis for important decision problems such as flood control, drought resisting, water resources utilization and so on. The main method is predicting changes of runoff through studying temperature, precipitation, evaporation, etc., which include two specific steps. Firstly, scenarios of the future climate changes are defined, and then the processes of hydrological cycle are simulated by using runoff forecasting techniques ( $\mathrm{Li}$ and Chen, 1999; Wang, 2006).

There are two common ways to set climate change scenarios. The first one is based on the atmospheric circulation model (GCM), and the second one is based on incremental scenario for probable changes of regional climate. Because GCM with a lot of uncertainty can not give a credible prediction of regional climate change (Jiang et al., 2004), incremental scenario method was used in this paper.

Medium and long-term runoff forecasting is a major issue that people are concerned about. Under the influence of climate changes and human activities, runoff has become more complex and difficult to be described precisely (Arnell and Reynard, 1996; Gao et al., 2002; Roger et al., 2006). Previous researches on runoff prediction often used causal prediction method (Vivek, 2002; David et al., 2004; Jonathan et al., 2004; Fujihara et al., 2008; Susan et al., 2008) and statistical prediction method (Liu Chunzhen, 2004; Chen et al., 2009). The former method is a deterministic prediction model based on the studies on atmospheric circulation, long-term weather processes and underlying physical conditions of basins, however it is difficult to be used in practice. The latter method applies statistical methods and historical data to establish forecasting model, which includes timeseries method (Hong, 1999; Maria et al., 2006), multiple

Received date: 2009-03-09; accepted date: 2009-10-09

Foundation item: Under the auspices of National Natural Science Foundation of China (No. 50809004)

Corresponding author: JIANG Yan. E-mail: lirenjy@sohu.com; yanjiang@rcees.ac.cn

(C) Science Press and Northeast Institute of Geography and Agroecology, CAS and Springer-Verlag Berlin Heidelberg 2010 
regression analysis method (Jiang et al., 2004; Wang and $\mathrm{Hu}, 2007$ ) and so on.

Based on the grey correlation analysis, He et al. (1998) presented a mid-long term forecasting method to predict spring runoff of the Manas River. Wang et al. (2008) proposed a new method based on BP neural network theory and Markov theory. In order to accurately predict the runoff of the Changjiang (Yangtze) River, Yu et al. (2009) introduced a new model-the projection pursuit auto-regression model, in which, the prediction factors were determined by using the auto-correlation analysis technique. The time-series method has the advantage of a long forecast period, but it can not take full use of the factors that have significant impacts on the forecast, such as climate information, thus leading to the prediction uncertainty.

Wang and Huang (2003) established the correlation between runoff and precipitation, and then applied a modified BP model to daily-runoff forecasting for the Huaihe River. Wang et al. (2007) combined wavelet, chaos and multiple regression to predict daily runoff. Cao et al. (2009) firstly took Dahuofang Reservoir as the research object and used the principium of principal component analysis method to extract the comprehensive factors affecting runoff change, then simulated comprehensive factors by Logistic equation, and finally set up Dahuofang Reservoir mid-long term forecasting model by using linear multi-regression method. The linear multi-regression method takes all the factors into account, but it ignores the developmental process of the predicted element itself.

Taking the nonlinear nature of runoff system into account and according to the scenario schemes of the changes of temperature and precipitation, and for overcoming the shortcomings of the time-series analysis method and regression forecast method, this paper established a Nonlinear Mixed Regression Model (NMR) to analyze the impacts of temperature and precipitation changes on annual river runoff process, which is a practical method to be studied.

\section{Model Building}

Supposed $x_{1}, x_{2}, \ldots, x_{s}(s>1)$ are linearly independent variables, $y$ is a dependent variable. If there is the following formula: $y=b_{0}+b_{1} x_{1}+\ldots+b_{s} x_{s}+\varepsilon$, then it is called s-linear regression model, where, $s$ is the number of variables; $b_{0}, b_{1}, \ldots, b_{s}$ are multi-regression coefficients, and $\varepsilon$ is a random error.

Auto-regression model is a common time-series model on the promotion of regression model. For time series $\{y(t)\}$, if there is the formula: $y(t)=a_{0}+a_{1} y(t-1)$ $+a_{2} y(t-2)+\ldots+a_{t-p} y(t-p)+\varepsilon(t)$, then, it is called $p$-auto-regression model, where, $t$ is the serial number; $p$ is the auto-regression order; $a_{0}, a_{1}, \ldots, a_{t-p}$ are auto-regression coefficients and $\varepsilon(t)$ is a white noise sequence.

Multi-regression model and auto-regression model are two basic statistical models. Even though both the models can give a good description for many random situations, they can not give a good simulation of complex hydrological processes. For overcoming the shortcomings, it is necessary to combine the two models to form a mixed regression model. Jiang et al., (2004) established a linear mixed regression model to forecast annual runoff of Sanmenxia Hydrological Station, in which annual runoff was a auto-regression factor, precipitation and air temperature were regression factors.

For a system with multi-input and single output, supposed its input is $\left\{x_{1}\right\},\left\{x_{2}\right\}, \ldots,\left\{x_{\mathrm{s}}\right\}$ and output is $\{y\}, \mathrm{a}$ linear mixed regression model can be set up as follows:

$$
\begin{aligned}
y(t)= & a_{0}^{(0)}+a_{1}^{(0)} y(t-1)+a_{2}^{(0)} y(t-1)+\cdots+a_{q_{0}}^{(0)} y\left(t-q_{0}\right)+ \\
& a_{1}^{(1)} x_{1}(t)+a_{2}^{(1)} x_{1}(t-1)+\cdots+a_{q_{1}}^{(1)} x_{1}\left(t-q_{1}+1\right)+\cdots+ \\
& a_{1}^{(s)} x_{s}(t)+a_{2}^{(s)} x_{s}(t-1)+\cdots+a_{q_{s}}^{(s)} x_{s}\left(t-q_{s}+1\right)+\varepsilon(t)
\end{aligned}
$$

where $y(t)$ is the output at time $t ; a^{(0)}, a^{(1)}, \ldots, a^{(s)}$ are response functions, also known as regression coefficients; $q_{0}, q_{1}, \ldots, q_{s}$ are model orders, $q_{0}$ is auto-regression order and $q_{1}, q_{2}, \ldots, q_{s}$ are regression orders; $\varepsilon(t)$ is residual.

Runoff formation process is a complexly natural phenomenon with the interaction of various factors. In different time scales, the factors impacting the change of runoff are different. But overall, without considering the situation of human activities, the main factors affecting river runoff are temperature and precipitation. Therefore, the hydrological system can be seen as a system with multi-inputs and single output. In the global background of climate change, changes of precipitation and temperature cause runoff change, and the response shows a simple linear relationship. Liu Changming (2004) revealed the nonlinear change of runoff rate with rainfall intensity at micro scale through a lot of slope rainfall 
experiments. At the same time, he confirmed the nonlinear relationship of annual runoff with annual precipitation and temperature at macro scale by hydrological model.

In this study, the inputs are the previous annual runoff $\left\{Q(t-1), Q(t-2), \ldots, Q\left(t-q_{0}\right)\right\}$, precipitation $\left\{P(t), P(t-1), \ldots, P\left(t-q_{1}+1\right)\right\}$, temperature $\{T(t), T(t$ $\left.-1), \ldots, T\left(t-q_{2}+1\right)\right\}$ and the output is annual runoff $\{Q(t)\}$. The nonlinear mixed regression model is built as follows:

$$
\begin{aligned}
Q(t)= & f\left(Q(t-1), \ldots, Q\left(t-q_{0}\right), P(t), \ldots, P\left(t-q_{1}+1\right),\right. \\
& \left.T(t), \ldots, T\left(t-q_{2}+1\right)\right)+\varepsilon(t)
\end{aligned}
$$

where $f(\cdot)$ is a mixed regression function, which is a nonlinear function; $q_{0}, q_{1}, q_{2}$ are model orders, $q_{0}$ is the auto-regression order, and $q_{1}$ and $q_{2}$ are regression orders; and $\varepsilon(t)$ is residual.

\section{Model Resolving}

\subsection{Order determination}

The model parameters and orders are different in different systems. There are four methods to estimate model order (Wang et al., 2007): auto-correlation fixedorder method (ACF) or partial auto-correlation fixed-order method (PACF), residual variance figure fixed-order method, F-test fixed-order method and the best criteria function fixed-order method. The basic idea of residual variance figure fixed-order method is to define a function, which should consider not only the error between measured value and simulated value, but also the number of model parameters. The minimum value of the function is the most appropriate order.

Supposed $\{z\}$ is measured series, $\{\hat{z}\}$ is simulated series, $t$ is serial number, $N$ is series length, then, $(z-\hat{z})$ is model residual and $\sum_{t=1}^{N}(z(t)-\hat{z}(t))^{2}$ is sum of square of model residual, then residual variance estimation $\sigma_{a}^{2}$ is:

$$
\sigma_{a}^{2}=\frac{\text { sum of sequar of model residual }}{\text { number of series }- \text { number of parameters }}
$$

where the number of series is $N-q_{0}$ and the number of parameters is $q_{0}+q_{1}+q_{2}$.

The model order can be determined by the change character of $\sigma_{a}^{2}$. As the model order increases, the de- nominator decreases. In less-fit, numerator reduces fast and $\sigma_{a}^{2}$ decreases. In over-fit, numerator reduces slowly and $\sigma_{a}{ }^{2}$ increases. We choose the lowest point of $\sigma_{a}{ }^{2}$ as an optimal order of the model.

\subsection{Parameters estimation}

According to Kolmogorov theorem, any time series can be seen as a nonlinear input-output system approximately. Because neural network has the ability to approximate any nonlinear mapping through network training, we adopt neural network to solve the nonlinear function in Equation (2).

$$
\operatorname{Set}\left\{(x(t), y(t)) \mid x \in R^{m}, y \in R^{n}, t=1,2, \cdots, N\right\} \text { are } N
$$

sample sets, where $R$ is mapping space, $m$ and $n$ are the dimensions of variable $x$ and $y$, respectively. For discrete space sequence, neural network can achieve the nonlinear mapping from the input to the output, that is, it can find some kinds of mapping $f$ subjected to $R^{m} \rightarrow R^{n}$. Supposed $x_{i}(t)$ is the input sample $i$ at time $t ; \hat{y}_{k}(t)$ is the output sample $k$ at time $t ; w_{i j}$ is the weight from the node $i$ in the input layer to the node $j$ in the hidden layer; $v_{j k}$ is the weight from the node $j$ in the hidden layer to the node $k$ in the output layer; $\theta_{j}$ is the threshold of the node $j$ in the hidden layer; $\gamma_{k}$ is the threshold of the node $k$ in the output layer. Then the neural network model can be expressed as:

$$
\begin{gathered}
\hat{y}_{k}(t)=f\left(\sum_{j=1}^{u} v_{j k} \times j\left[\sum_{i=1}^{m} w_{i j} x_{i}(t)+\theta_{j}\right]+\gamma_{k}\right) \\
(k=1,2, \ldots, n ; t=1,2, \ldots, N)
\end{gathered}
$$

where $f$ is the sigmoid function, $f(x)=1 /\left(1+\mathrm{e}^{-x}\right) ; \varphi$ is the activation function from the input layer to the hidden layer. Supposed the total error $(E)$ is less than $\varepsilon$, that is, $E=\frac{1}{2} \sum_{t=1}^{p} \sum_{k=1}^{n}\left[y_{k}(t)-\hat{y}_{k}(t)\right]^{2} \leq \varepsilon$. We use optimization algorithm to resolve the network. If $E$ is less than $\varepsilon$, the network training is completed.

\section{Model Forecasting}

According to the above model structure, a runoff forecast model was set up using data of annual precipitation, annual temperature and annual runoff from Daiying Hydrological Station in Hebei Province, China in 1956-2000. The first 37-year data were used as training samples and the last 8-year data as verified samples. 


\subsection{Study area}

The Chaohe River Basin $\left(40^{\circ} 20^{\prime}-41^{\circ} 27^{\prime} \mathrm{N}, 116^{\circ} 87^{\prime}-117^{\circ}\right.$ $34^{\prime} \mathrm{E}$ ) was chosen as a study area. The river flows past Fengning County and Luanping County of Hebei Province, China, and flows into the Miyun Reservoir after converging with its tributary of the Andaomuhe River. The area of the basin is $4787 \mathrm{~km}^{2}$, and the length of the river is about $170 \mathrm{~km}$. The elevation of the basin is between $200 \mathrm{~m}$ and $2194 \mathrm{~m}$, with an average elevation of $851 \mathrm{~m}$. The average longitudinal gradient is $8.4 \%$. Daiying Hydrological Station is a control station for the Chaohe River Basin. The area of catchment upstream from the control section is $4701 \mathrm{~km}^{2}$, which accounts for $99.9 \%$ of Chaohe River Basin. The climate of the study area belongs to the temperate continental monsoon climate. It is cold and dry in winter, and rainy and hot in summer. And it is also characterized by great diurnal temperature amplitude, frequent strong winds and long sunshine hours. The average annual temperature is $5^{\circ} \mathrm{C}-8{ }^{\circ} \mathrm{C}$, and the maximum is $41.5^{\circ} \mathrm{C}$ and the minimum is $-29.5^{\circ} \mathrm{C}$. The average annual precipitation is $528 \mathrm{~mm}$, of which $70 \%-80 \%$ happens in flood season between June and September. Moreover, the interannual variation of precipitation is greater, with a maximum being 2.3 times as much as the minimum. The average annual evaporation is $916 \mathrm{~mm}$, and the aridity index is 1.73 . The average annual runoff flow is $18.04 \times 10^{9} \mathrm{~m}^{3}$, and the annual average runoff depth is $74.7 \mathrm{~mm}$.

\subsection{Data handling}

At first, data are standardized to eliminate dimensionless. Set the mean and variance of the original sequence $\{x\}$ are $\bar{x}$ and $\sigma$, then the standardized sequence $\{y\}$ can be expressed as:

$$
y=(x-\bar{x}) / \sigma
$$

Because neural network uses s-shaped function in range of $[0,1]$ as the transfer function, the standardized data need to be normalized to improve the accuracy of prediction. In this paper, we normalize the data to range $[0.1,0.9]$, and the normalized formula is:

$$
y^{\prime}=0.1+0.8\left(y-y_{\min }\right) /\left(y_{\max }-y_{\min }\right)
$$

where $\left\{y^{\prime}\right\}$ is the normalized series, and $y_{\max }$ and $y_{\min }$ are the maximum and minimum of data, respectively.

Then the predicted data need to be restituted. The reductive formula is:

$$
x=\bar{x}+\sigma\left[y_{\text {min }}+1.2\left(y^{\prime}-0.1\right)\left(y_{\text {max }}-y_{\text {min }}\right)\right]
$$

\subsection{Determination of model parameters}

Given different auto-regression orders and regression orders in the nonlinear mixed regression model (Equation (2)), the fixed-order formula (Equation (3)) is used to calculate residual variance of the normalized data. The results are shown in Table 1.

Table 1 Calculated results of residual variance

\begin{tabular}{cccc}
\hline No. & $\begin{array}{c}\text { Order } \\
\left(q_{0}, q_{1}, \mathrm{q}_{2}\right)\end{array}$ & $\begin{array}{c}\text { Sum of squares of } \\
\text { residual }\end{array}$ & $\begin{array}{c}\text { Residual variance } \\
\left(\sigma_{a}^{2}\right)\end{array}$ \\
\hline 1 & $(1,1,1)$ & 0.7087 & 0.0173 \\
2 & $(1,1,2)$ & 0.6581 & 0.0165 \\
3 & $(1,1,3)$ & 0.6486 & 0.0166 \\
4 & $(1,2,1)$ & 0.6648 & 0.0166 \\
5 & $(1,3,1)$ & 0.6541 & 0.0168 \\
6 & $(2,2,2)$ & 0.5623 & 0.0148 \\
7 & $(3,2,2)$ & 0.5568 & 0.0150 \\
8 & $(3,3,2)$ & 0.5210 & 0.0153 \\
9 & $(3,3,3)$ & 0.5076 & 0.0154 \\
\hline
\end{tabular}

From Table 1, we can see that when both the auto-regression order and regression order are 2, we can get the smallest residual variance. Therefore, the factors affecting future runoff include precipitation, temperature, as well as pre-precipitation, pre-temperature and pre-runoff. According to Kolmogorov theorem, we determine the nodes of hidden layer of the BP neural network model 13 and select the network structure $[6,13,1]$ to forecast annual runoff.

In BP neural network, we select tansig function as activation function of hidden layer and logsig function as activation function of output layer. Traingdx function is used to train network. Acceptable error, a maximum number of iteration and study efficiency are set 0.01 , 5000 and 0.1 , respectively.

\subsection{Analyses of forecasting results}

In this paper, we use BP neural network to calibrate nonlinear mixed regression model and get the weights and threshold, with the first 37-year data, starting from 1956. Then the last 8-year data are used to verify the accuracy of the model. The simulation results show that water balance error can be controlled in the allowed range (no more than 5\%), and the model efficiencies in the periods of calibration and verification are 0.82 and 
0.76 , respectively (Table 2 ). The results indicate that the nonlinear mixed regression model can forecast annual runoff effectively.

Table 2 Simulating results for Daiying Hydrological Station in periods of calibration and verification

\begin{tabular}{ccccc}
\hline Period & $\begin{array}{c}\text { Observed } \\
\text { annual runoff } \\
\left(\times 10^{6} \mathrm{~m}^{3}\right)\end{array}$ & $\begin{array}{c}\text { Simulated } \\
\text { annual runoff } \\
\left(\times 10^{6} \mathrm{~m}^{3}\right)\end{array}$ & $\begin{array}{c}\text { Relative } \\
\text { error } \\
(\%)\end{array}$ & $\begin{array}{c}\text { Model } \\
\text { efficiency }\end{array}$ \\
\hline $1958-2000$ & 273 & 265 & -2.87 & 0.81 \\
$1958-1992$ & 275 & 268 & -2.26 & 0.82 \\
$1993-2000$ & 264 & 251 & -4.59 & 0.76 \\
\hline
\end{tabular}

Figures $1-4$ show the runoff simulating results and the scatter plots in the period of calibration and verification, respectively. From these figures, we can see that whether in the period of calibration or verification, the errors between predicted runoff and observed runoff are small. Therefore, we can arrive at the result that the proposed method can simulate the observed runoff and has good prediction performance.

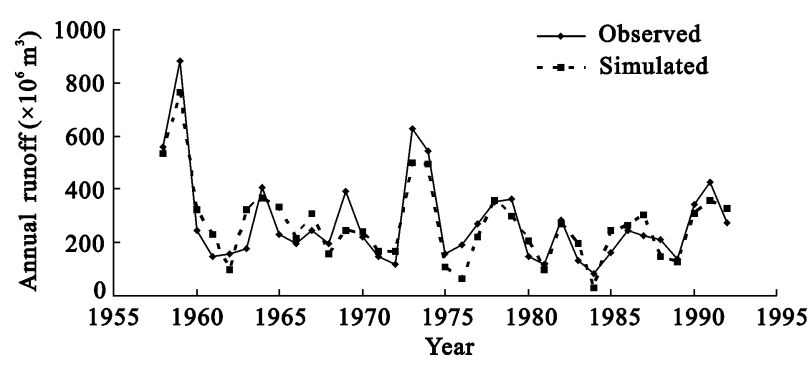

Fig. 1 Runoff simulating results in the period of calibration

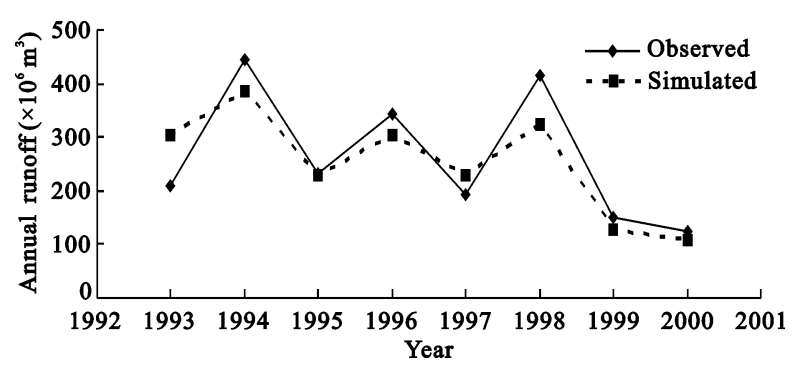

Fig. 2 Scatter plot in the period of verification

To evaluate the superiority of Nonlinear Mixed Regression Model (NMR), Auto-regression Model (AR), Multi-Regression Model (MR) and Linear Mixed Regression Model (LMR) are used for comparison for the same numerical example. From Table 3 we can see that the NMR based on BP neural network has the highest forecasting qualified ratio $75 \%$. And at the same time, it

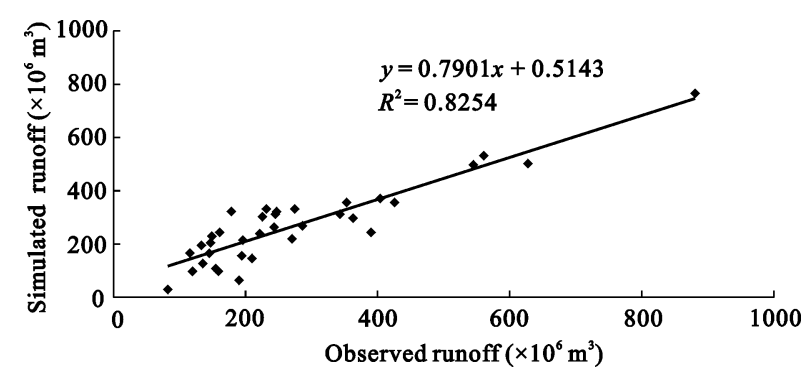

Fig. 3 Runoff simulating results in the period of calibration

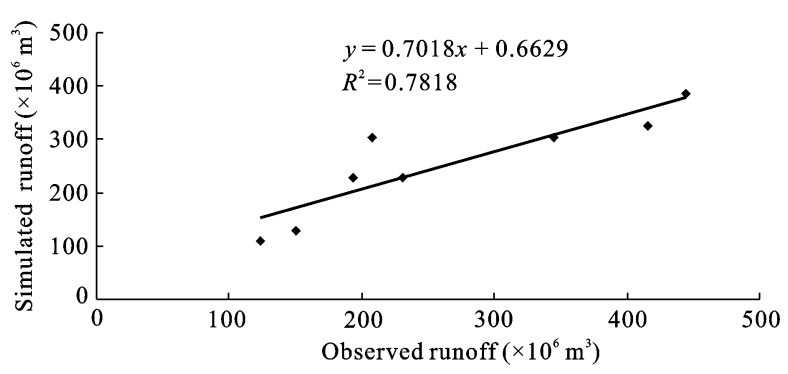

Fig. 4 Scatter plot in the period of verification

has the smallest relative error. The proposed method reduces the scope of error variation and has more stable forecasting and high precision. Therefore, it is efficient and effective for annual runoff forecast and can provide more scientific basis for the development of hydroelectric power generation plans.

\section{Scenario Simulation}

In order to provide reliable hydrological data for hydraulic engineering, this paper researches the future annual runoff change of Daiying Hydrological Station under the global climate change. The average annual temperature, precipitation and runoff of the station were $6.73^{\circ} \mathrm{C}$, $474.29 \mathrm{~mm}$ and $273 \times 10^{6} \mathrm{~m}^{3}$ in 1958-2000. For analyzing the impact of precipitation and temperature changes on runoff, according to climate change trends of recent decades, we use scenario method to forecast runoff in condition of temperature changes of $\pm 1^{\circ} \mathrm{C}, \pm 2^{\circ} \mathrm{C}$ and $\pm 3^{\circ} \mathrm{C}$, and precipitation changes of $\pm 20 \%, \pm 40 \%, \pm 60 \%, \pm 80 \%$ and $\pm 100 \%$. The results are shown in Table 4 .

As seen from Table 4, when the precipitation remains unchanged, if the temperature increases $1{ }^{\circ} \mathrm{C}, 2{ }^{\circ} \mathrm{C}$ and $3^{\circ} \mathrm{C}$, the average annual runoff will reduce by $6.83 \%$, $13.53 \%$ and $21.23 \%$, respectively. In contrast, when the temperature remains unchanged, if the precipitation decreases by $20 \%, 40 \%, 60 \%$ and $80 \%$, the average annual runoff will decrease by $44.76 \%, 56.00 \%, 74.55 \%$ and 
Table 3 Comparison of AR, MR, LMR and NMR

\begin{tabular}{|c|c|c|c|c|c|c|c|c|c|}
\hline \multirow{2}{*}{ Year } & \multirow{2}{*}{$\begin{array}{l}\text { Observed annual runoff } \\
\qquad\left(\times 10^{6} \mathrm{~m}^{3}\right)\end{array}$} & \multicolumn{4}{|c|}{ Simulated annual runoff $\left(\times 10^{6} \mathrm{~m}^{3}\right)$} & \multicolumn{4}{|c|}{ Relative error $(\%)$} \\
\hline & & $\mathrm{AR}$ & MR & LMR & NMR & $\mathrm{AR}$ & MR & LMR & NMR \\
\hline 1993 & 208 & 207 & 158 & 178 & 304 & -0.48 & -24.03 & -14.42 & 46.15 \\
\hline 1994 & 444 & 272 & 260 & 266 & 385 & -38.74 & -41.44 & -40.09 & -13.29 \\
\hline 1995 & 231 & 371 & 299 & 280 & 228 & 60.61 & 29.44 & 21.21 & -1.30 \\
\hline 1996 & 345 & 141 & 370 & 371 & 304 & -59.13 & 7.25 & 7.54 & -11.88 \\
\hline 1997 & 193 & 361 & 092 & 162 & 228 & 87.05 & -52.33 & -16.06 & 18.13 \\
\hline 1998 & 416 & 179 & 514 & 506 & 324 & -56.97 & 23.56 & 21.63 & -22.12 \\
\hline 1999 & 150 & 384 & 83 & 120 & 128 & 156.00 & -44.67 & -20.00 & -14.67 \\
\hline 2000 & 123 & 115 & 88 & 91 & 108 & -6.50 & -28.46 & -26.02 & -12.20 \\
\hline Qualified ratio & & & & & & 25.00 & 12.50 & 50.00 & 75.00 \\
\hline
\end{tabular}

Table 4 Annual runoff change under climate change (\%)

\begin{tabular}{ccccccccccccc}
\hline \multirow{2}{*}{ Temperature change $\left({ }^{\circ} \mathrm{C}\right)$} & \multicolumn{10}{c}{ Precipitation change (\%) } \\
\cline { 2 - 12 } & -100 & -80 & -60 & -40 & -20 & 0 & 20 & 40 & 60 & 80 & 100 \\
\hline-3.0 & -100 & -84.64 & -61.46 & -42.27 & -25.12 & 21.12 & 67.58 & 113.93 & 160.28 & 206.62 & 252.79 \\
-2.0 & -100 & -86.67 & -65.91 & -46.91 & -31.65 & 14.05 & 60.29 & 106.02 & 151.56 & 197.35 & 242.70 \\
-1.0 & -100 & -88.40 & -70.18 & -51.73 & -38.35 & 9.15 & 53.27 & 98.65 & 143.37 & 188.17 & 233.07 \\
0 & -100 & -90.93 & -74.55 & -56.00 & -44.76 & -0.52 & 46.35 & 88.65 & 134.56 & 178.53 & 222.79 \\
1.0 & -100 & -93.39 & -78.73 & -60.19 & -50.65 & -6.83 & 39.22 & 81.02 & 126.01 & 169.44 & 212.97 \\
2.0 & -100 & -95.84 & -82.64 & -64.73 & -56.05 & -13.53 & 32.19 & 73.83 & 116.63 & 160.81 & 203.34 \\
3.0 & -100 & -97.93 & -86.56 & -69.37 & -60.58 & -21.23 & 25.12 & 66.92 & 107.91 & 151.35 & 194.25 \\
\hline
\end{tabular}

$90.93 \%$, respectively; and if precipitation increases by $20 \%, 40 \%, 60 \%, 80 \%$ and $100 \%$, the average annual runoff will increase by $46.35 \%, 88.65 \%, 134.56 \%$, $178.53 \%$ and $222.79 \%$, respectively.

Therefore, we can arrive at the following conclusions. The annual runoff has a different percentage of increase or decrease with the temperature changing. If temperature increases, the annual runoff will decrease. In contrast, if temperature decreases, the annual runoff will increase. The reason is that temperature rising increases evaporation. Changes of precipitation also influence changes of annual runoff. If precipitation increases, the annual runoff will increase, and the increase rate of runoff is larger than that of precipitation. In contrast, if precipitation decreases, the annual runoff will decrease. Similarly, the decrease rate of runoff is smaller than that of precipitation.

\section{Conclusions}

In this paper, Nonlinear Mixed Regression Model (NMR) was established by taking the nonlinear nature of runoff system into account, and combining auto-regression method and multi-regression method, to analyze the impact of temperature and precipitation changes on annual river runoff process. The model was calibrated and verified by using BP neural network with observed meteorological and runoff data from Daiying Hydrological Station on the Chaohe River of Hebei Province in 1956-2000. Model efficiency in the periods of calibration and verification is 0.82 and 0.76 , respectively. The results indicate that the proposed model can forecast annual runoff effectively. Comparing with auto-regression model, linear multi-regression model and linear mixed regression model, NMR can remarkably improve forecasting precision. Moreover, NMR was used to forecast runoff of Daiying Hydrological Station in the Chaohe River Basin under a variety of climate changes. The scenario simulation results show that NMR method can overcoming the disadvantages of $\mathrm{AR}$ and MR, and can give full consideration to the nonlinear relationship of hydrological processes. Because the study area is a typical arid region in the northern China, the proposed model has important practical value for water resources research in arid regions, which also needs to be further studied. 


\section{References}

Arnell N W, Reynard N S, 1996. The effects of climate change due to global warming on river flows in Great Britain. Journal of Hydrology, 183(3-4): 397-424. DOI: 10.1016/0022-1694 (95)02950-8

Cao Yongqiang, You Hailin, Xing Xiaosen et al., 2009. Multiple regression runoff forecasting model based on logistic equation and its application. Water Power, (6): 12-14. (in Chinese)

Chen Yaning, Xu Changchun, Hao Xingming et al., 2009. Fifty-year climate change and its effect on annual runoff in the Tarim River Basin, China. Quaternary International, 208(1-2): 53-61. DOI: 10.1016/j.quaint.2008.11.011

David L, Yves G, Jean L P et al., 2004. Evidence for global runoff increase related to climate warming. Advances in Water Resources, 27(6): 631-642. DOI: 10.1016/j.advwatres.2004.02.020

Fujihara Y, Tanaka K, Watanabe T et al., 2008. Assessing the impacts of climate change on the water resources of the Seyhan River Basin in Turkey: Use of dynamically downscaled data for hydrologic simulations. Journal of Hydrology, 353(1-2): 33-48. DOI: $10.1016 /$ j.jhydrol.2008.01.024

Gao S, Wang J, Xiong L et al., 2002. A macro-scale and semidistributed monthly water balance model to predict climate change impacts in China. Journal of Hydrology, 268(1): 1-15. DOI: 10.1016/S0022-1694(02)00075-6

He Xilin, Dong Xianjun, Zhou Jianwei, 1998. A grey forecasting method of mid-long term spring runoff forecasting in Manas River. Journal of Shihezi University (Natural Science), 2(3): 227-230. (in Chinese)

Hong Shizhong, 1999. Nonlinear time series analysis and its application to geoscience. Advance in Earth Sciences, 14(6): 559-565. (in Chinese)

Jiang Xiaohui, Liu Changming, Liu Yu et al., 2004. Mix regression model application for forecast annual flow in Yellow River under duality model. Science in China (Ser. E), 34(supp.): 95-102. (in Chinese)

Jonathan I M, Graciana P, Kenneth M M, 2004. Evaluation of the impact of climate change on hydrology and water resources in Swaziland: Part II. Physics and Chemistry of the Earth, 29(1518): 1193-1202. DOI: 10.1016/j.pce.2004.09.035

Li Kerang, Chen Yufeng, 1999. The progress in methodologies to study impacts of global climate change in China. Geographical Research, 18(2): 214-219. (in Chinese)
Liu Changming, 2004. Study of some problems in water cycle changes of the Yellow River basin. Advances in Water Science, 15(5): 608-614. (in Chinese)

Liu Chunzhen, 2004. The issue in the study of climate change on the terrestrial hydrological cycle. Advances in Earth Science, 19(1): 115-119. (in Chinese)

Maria H, Martin F, Michael S et al., 2006. An application of artificial neural networks to carbon, nitrogen and phosphorus concentrations in three boreal streams and impacts of climate change. Ecological Modelling, 195(1-2): 51-60. DOI: 10.1016/j.ecolmodel.2005.11.009

Roger N J, Francis H S, Walter C et al., 2006. Estimating the sensitivity of mean annual runoff to climate change using selected hydrological models. Advances in Water Resources, 29(10): 1419-1429. DOI: 10.1016/j.advwatres.2005.11.001

Science U N A O, 1977. Climate Change and Water Supply. Washington D C: National Academy Press.

Susan S D, PeterL, Ray M et al., 2008. The impacts of climate change on hydrology in Ireland. Journal of Hydrology, 356(1-2): 28-45. DOI: 10.1016/j.jhydrol.2008.03.025

Vivek K A, 2002. The use of the aridity index to assess climate change effect on annual runoff. Journal of Hydrology, 265(1-4): 164-177. DOI: 10.1016/S0022-1694(02)00101-4

Wang Ling, Huang Guoru, 2003. Application of modified BP model to basin daily-runoff forecasting. Water Resources and Power, 21(1): 32-34. (in Chinese)

Wang Shunjiu, 2006. Impacts of global climate change on hydrology and water resources. Advances in Climate Change, 2(5): 223-227. (in Chinese)

Wang Xiujie, Lian Jijian, Fei Shouming et al., 2007. Chaotic multivariate autoregressive model of daily runoff prediction based on wavelet de-noising. Journal of System Simulation, 19(15): 3605-3608. (in Chinese)

Wang Yimin, Yu Xingjie, Chang Jianxia, 2008. Prediction of runoff based on BP neural network and Markov model. Engineering Journal of Wuhan University, 41(5): 14-18. (in Chinese)

Wang Zhenglong, $\mathrm{Hu}$ Yonghong, 2007. Applied Time Series Analysis. Beijing: Science Press. (in Chinese)

Yu Guorong, Ye Hui, Xia Ziqiang et al., 2009. Application of projection pursuit auto-regressionmodel in predicting runof of Yangtze River. Journal of Hohai University (Natural Sciences), 37(3): 263-266. (in Chinese) 